\title{
COVID-19 in patients with chronic inflammatory rheumatic diseases: Do these patients have a higher risk of COVID-19 due to their underlying medication?
}

\author{
Claudia Dragomir', Alexandra Maria Burlui',2, Geanina-Florica Popescu', \\ Ioan-Teodor Dragoi', Anca Cardoneanu ${ }^{1,2}$, Luana Andreea Macovei ${ }^{1,2}$, Elena Rezus ${ }^{1,2}$ \\ ${ }^{1}$ Reumatology I Clinic, Clinical Rehabilitation Hospital, lasi, Romania \\ ${ }^{2}$ "Grigore T. Popa“ University of Medicine and Pharmacy, Iasi, Romania
}

\begin{abstract}
Since the first case was reported to the World Health Organization (in late December 2019 in Wuhan, Hubei Province, China), by mid-June 2021, there have been 175,541,600 confirmed cases of COVID-19 globally, including 3,798,361 reported deaths. Many of those infected with SARS-CoV-2 have an asymptomatic form of the disease or mild flu-like symptoms. In another category of patients, the disease may be more aggressive, with a severe form that can lead to acute respiratory distress syndrome (ARDS), respiratory failure, and even death with a negative impact on patients.

The COVID-19 pandemic has arisen serious concerns in the rheumatology community regarding the management of immunosuppressed patients diagnosed with inflammatory rheumatic diseases. It has been stated that severe forms of COVID-19 occur as a result of exacerbated inflammation status and cytokine production. Nevertheless, it remains unclear whether the use of biological agents subjects the patient to a higher risk, or rather protects them against severe forms of the disease.

According to the American College of Rheumatology, rheumatic diseases were not identified as a risk factor that predicted poor outcome in patients with COVID-19. However, various studies have certified that corticosteroid treatment in patients with chronic rheumatic diseases at a dose $>10 \mathrm{mg} /$ day is associated with an increased risk of infection as well as the possibility of developing more severe COVID-19 symptoms.
\end{abstract}

Keywords: COVID-19 disease, immune-mediated rheumatic diseases, health behaviour, DMARDs, corticosteroids, autoantibodies, comorbidities

\section{INTRODUCTION}

Severe acute respiratory syndrome coronavirus 2 (SARS-CoV-2) is part of the Coronaviridae family, genus Coronavirus and is a novel beta coronavirus that belongs to the same subgenus as the Severe Acute Respiratory Syndrome Coronavirus (SARS-CoV) and the Middle East Respiratory Syndrome Coronavirus (MERS-CoV) (1). The name derives from the Greek

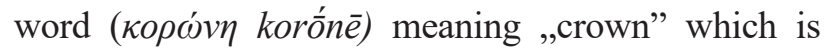
referring to the corona-like appearance while observed under an electron microscope (2-4). SARS-CoV and MERS-CoV have been previously implicated in SARS-CoV epidemics (during the winter of 2002 to
2003, the last known case being recorded in mid-2004) with 10\% mortality and MERS-CoV epidemics (first identified in Saudi Arabia in 2012) with mortality rates up to $35 \%(3-5)$.

On March 11th 2020, the World Health Organization (WHO) declared the coronavirus-19 (COVID-19) outbreak a global pandemic, causing an unprecedented global health crisis $(3,6,7)$. The first case of COVID-19 was reported to the WHO in Wuhan, Hubei Province, China, in late December 2019 (8), and since the first case, by mid-June 2021, there have been 175,541,600 confirmed cases of COVID-19 globally, including $3,798,361$ reported deaths $(6,7)$. COVID-19 may cause 
flu-like symptoms, anosmia, ageusia or even thrombotic complications (9). In another category of patients, SARS-CoV-2 infection can lead to acute respiratory distress syndrome (ARDS), together with various other manifestations (10). Most patients showed signs of disease after an incubation period of 1-14 days $(3,10,11)$.

\section{ARE CHRONIC INFLAMMATORY RHEUMATIC DISEASES ASSOCIATED WITH AN INCREASED RISK OF COVID-19 INFECTION?}

Chronic inflammatory rheumatic diseases constitute a heterogeneous group of multisystem autoimmune disorders (including rheumatoid arthritis - RA, spondyloarthrites - SpA, systemic vasculitides, as well as connective tissue diseases - CTD such as systemic lupus erythematosus - SLE, systemic sclerosis - SSc, mixed connective tissue disease - MCTD, and Sjögren syndrome - SjS) (12). These conditions have all been associated with an increased risk of morbidity and mortality and require multidisciplinary management together with chronic disease-modifying treatment (13).

In early 2020, at the onset of the COVID-19 pandemic, many concerns have been raised regarding COVID-19 in patients with immune-mediated inflammatory diseases, which affect an estimated 4-5\% of the global population $(14,15)$. These patients are known to be at higher risk of severe infections, not only due to their underlying immune dysfunction but also as a consequence of immunosuppressant therapy $(14,16,17)$.

The immune dysregulation that has been associated with the pathogenesis of immune-inflammatory rheumatic diseases together with the medications used in their management impact both innate immune responses (which play a critical role in viral infections), as well as the adaptive immune responses through intricate mechanisms (18). In the early stages of COVID-19, failure to reduce the viral load may result in an exaggerated inflammatory reaction leading to tissue damage and multiple organ involvement $(15,18)$.

During the progression of the COVID-19 pandemic, numerous questions were raised regarding patients with autoimmune diseases under certain medications (disease-modifying or symptomatic treatment), with a need for further investigation if they were found to be more likely to contract the virus and develop COVID-19. Some questions have also arisen about the disease form these patients would develop, more specifically if they could have a more aggressive form of the disease compared to the general population $(14,15,19)$.

Several studies showed that patients with chronic inflammatory diseases may be at higher risk for a more severe course of COVID-19, including longer hospitalization or the development of life-threatening complications $(20,21)$. Similar to the general population, patients with rheumatic conditions and cardiovascular comorbidities have been shown to be at increased risk for COVID-19-related complications $(10,21)$. Nevertheless, in a retrospective multicenter cohort study which included 6792 patients with PCRconfirmed (polymerase chain reaction) COVID-19, Ungaro et al. demonstrated that immune-inflammatory rheumatic diseases were not an independent risk factor for severe forms of COVID-19 $(22,23)$.

Another study investigated outcomes in 52 patients with inflammatory rheumatic conditions and 104 who had not been diagnosed with an autoimmune rheumatic disorder. The authors reported that patients with chronic immune-inflammatory rheumatic conditions did not demonstrate a higher mortality risk due to COVID-19 $(22,23)$.

According to the American College of Rheumatology (ACR) who formed a COVID-19 clinical task force on March 26th 2020, rheumatic diseases were not identified as a risk factor for poor outcomes in patients with COVID-19 (24).

The risk factors that predict poor outcomes in patients with COVID-19 include age $>65$ years, obesity, hypertension, diabetes mellitus, chronic lung disease, cardiovascular disease (CVD) and chronic kidney disease (CKD) (25).

\section{DO PATIENTS WITH CHRONIC RHEUMATIC DISEASES HAVE A HIGHER RISK OF COVID-19 DUE TO THE UNDERLYING MEDICATION?}

The main objective of the treatment used in chronic rheumatic conditions is to obtain remission or low disease activity, while also aiming to hamper the radiological progression, reduce pain, prevent organ damage, and maintain joint function (26).

Data resulting from observational research are discrepant, some studies suggesting that patients receiving chronic treatment for immune-inflammatory rheumatic diseases may be at increased risk of developing COVID-19 and its complications, while others indicate either a similar or a decreased risk compared to persons not undergoing the above-mentioned therapies $(20,28)$. 


\section{Non-steroidal anti-inflammatory drugs (NSAIDs)}

During the course of viral infections, the role of NSAIDs remains controversial. For example, ibuprofen could theoretically worsen the clinical course and increase susceptibility to COVID-19 because of its ability to induce the overexpression of ACE2 (angiotensin-converting enzyme 2) (29).

In the context of the COVID-19 pandemic, the WHO initially stated that the use of NSAIDs may be risky in patients with COVID-19. This warning was based on a study of ibuprofen use in pneumonia patients in France (30). After gathering further data on the use of NSAIDs in patients with confirmed SARSCoV-2 infection, the WHO stated that there is currently no evidence that NSAID use could lead to severe adverse events or acute healthcare utilization in patients with COVID-19 (31).

The ACR guidelines state that NSAIDs may be initiated in patients with active arthritis, yet in persons with documented or suspected COVID-19 who present with severe respiratory symptoms, NSAIDs should be stopped (24). In the absence of severe respiratory symptoms, there is no clear recommendation to discontinue NSAIDs.

The Australian Rheumatology Association (ARA) and the National Institute for Health and Care Excellence (NICE) guidelines state that patients taking NSAIDs for an inflammatory rheumatic disease such as RA may continue treatment even in the context of SARS CoV-2 infection (31).

In a study including 3,136 patients with inflammatory rheumatic diseases, NSAID treatment effects were observed in 362 patients, of which 12 developed COVID-19 (16). Furthermore, regarding hospitalization rates in patients with immune-mediated rheumatic diseases and COVID-19, NSAID use was not associated with hospitalization status. Moreover, there was no negative effect of NSAIDs on clinical outcomes (20). It has been stated that there is no correlation between NSAIDs and the worsening of COVID-19 symptoms in patients with immune-inflammatory rheumatic conditions (32).

COVID-19-related mortality among persons with chronic inflammatory rheumatic diseases was studied by Strangfeld et al. A total of 3,729 patients were included, 638 taking NSAIDs. The results reported by the authors did not indicate that NSAIDs have a negative role in the outcome of patients with inflammatory rheumatic diseases diagnosed with COVID-19 (33).

\section{Corticosteroids}

According to the currently available data, the issue of determining whether or not corticosteroids bring notable benefits in the treatment of COVID-19 remains a matter of debate (34). In autoimmune rheumatic diseases, corticosteroids are frequently used due to their anti-inflammatory effects. However, glucocorticoid therapy has been associated with a significant risk of infection. The COVID-19 pandemic posed challenges to both clinicians and researchers regarding the management of these cases, including the safety of corticoid use. Nevertheless, it has been stated that corticosteroid treatment may be a risk factor for SARS CoV-2 infection and could increase the odds of developing a severe form of COVID-19. Prolonged courses of prednisone $>10 \mathrm{mg} /$ day have been associated with an increased risk of developing a severe form of COVID-19 (20).

The British Society of Rheumatology (BSR) states that patients receiving low-dose corticosteroids together with immunosuppressant therapy and patients receiving high-dose steroids (>20 mg/day) are part of a high-risk group for COVID-19 (35). A higher risk of hospitalization in patients with inflammatory arthritis taking glucocorticoids was also observed in a published case series from New York (28). The median daily dose of prednisone was associated with the development of COVID-19 (16). Furthermore, an increased risk of mortality due to COVID-19 was linked with higher doses of corticosteroids (36).

The COVID-19 Global Rheumatology Alliance (COVID-19 GRA) patient registry showed that the most common rheumatic disease in which the infection with documented SARS-CoV2 infection was RA (694 RA of 1,783 patients) (31). Moreover, RA has been associated with an increased risk of COVID-19 compared to the general population. This risk is thought to be related to several factors that are common for RA and COVID-19 (37). Several studies examined the relationship between corticoid therapy and COVID-19 outcomes. Recent research conducted in Iran reported 10 cases of RA who had COVID-19. Of these patients, those receiving corticosteroids had a more severe form of the disease $(38,39)$.

It has been stated that CTD or vasculitis could represent risk factors for COVID-19. Patients with RA and SLE who were treated with immunosuppressive medication and had low disease activity or were in remission had a lower risk of contracting the dis- 
ease compared to those with high disease activity. It has been suggested that the risk of infection may be increased by disease flares and corticosteroid use (37). Patients with SLE using glucocorticoids at a dose $>10 \mathrm{mg}$ per day were found to be at higher risk of severe COVID-19 $(38,40,41)$.

A French patient with granulomatosis with polyangiitis (GPA), who was on corticosteroid treatment and Rituximab (RTX), had severe COVID-19 and required intubation due to acute respiratory distress syndrome. However, due to the known respiratory tract involvement in GPA as well as the concomitant treatment with RTX, it is unlikely that glucocorticoid therapy was the only risk factor for severe COVID-19 in this case (42).

\section{Disease-modifying antirheumatic drugs (DMARDs)}

It has been stated that synthetic or biological disease-modifying antirheumatic drugs (DMARDs) are not linked to COVID-19-related deaths $(33,43)$. Nevertheless, a study performed by COVID-19 GRA which included a new primary outcome (COVID-19related deaths) and the use of an active medication comparator (Methotrexate - MTX monotherapy) examined 3729 patients with chronic inflammatory rheumatic diseases $(6,27,20)$. It was found that treatment with Sulfasalazine (SSZ) and immunosuppressants (including Azathioprine - AZA, Cyclophosphamide CYC, Cyclosporine A - CsA, mycophenolate mofetil - MMF, and Tacrolimus - TAC) were related to higher odds of death compared to MTX monotherapy, yet there were no higher odds of death with other DMARDs $(6,20,27)$. Moreover, the available data from the COVID-19 GRA registry stated that treatment with Leflunomide (LEF), antimalarials (Hydroxychloroquine - HCQ), Tumor Necrosis Factor $\alpha$ inhibitors (TNFi), Abatacept (ABA), Belimumab (BLM), IL-6 inhibitors, IL-17/IL-23/IL-12+23 inhibitors and targeted synthetic (tsDMARDs) were not associated with a higher mortality risk compared to MTX monotherapy (33).

In a multicentre cross-sectional study (3028 patients with inflammatory rheumatic diseases recruited from six countries), the underlying treatment with DMARDs was associated with a reduced occurrence of COVID-19 (16).

Furthermore, available data from the COVID-19 GRA registry highlights that treatment with bDMARDs or tsDMARD monotherapy prior to COVID-19 diagnosis was significantly associated with lower risk of hospitalization compared to patients without DMARD therapy (20). Moreover, underlying treatment with csDMARDs alone or in combination with biologics or Janus Kinase inhibitors (JAKi) in patients with CTD was associated with a decreased rate of hospitalization $(20,44)$. A recent retrospective study included 47 patients of which $53.2 \%$ were diagnosed with RA, followed by SLE (21.3\%) and PsA (10.6\%). Among the study group, 93.6\% were receiving DMARDs, yet almost $60 \%$ of the patients stopped medication due to COVID-19 (44).

Initially, it was thought that many therapies used in the treatment of immune-inflammatory rheumatic diseases were associated with a higher risk of COVID19-related complications. Nevertheless, several studies have shown that mice deficient in $\mathrm{TNF} \alpha$ or its receptor were protected from a severe form of COVID-19 $(7,45)$. Furthermore, TNF $\alpha$ levels are elevated in patients with COVID-19 42 (it is known that increased levels of TNF $\alpha$ promote the expression of ACE2 which enhances viral entry into cells) (46). Therefore, therapies targeting this pathway (TNFi) have been proposed to possibly treat patients with severe COVID-19 (47). Patients with inflammatory rheumatic diseases which receive TNFi do not seem to have an increased risk of COVID-19 (48).

Recently published work considered the possibility that therapeutic agents targeting B cells (such as RTX, able to deplete B-lymphocyte populations) may lead to a higher risk of COVID-19 or its complications $(7,49)$.

However, scientific data on this matter remain discrepant. Certain studies do not confirm the above-mentioned hypothesis, patients with immune-mediated diseases treated with anti-CD20 agents (cluster of differentiation 20) not demonstrating an increased risk of severe COVID-19 (7). Nevertheless, according to a retrospective study, patients with inflammatory rheumatic diseases who were treated with RTX developed more severe forms of COVID-19 and required a longer hospital stay $(50,51)$.

A case report of an ANCA-associated (anti-neutrophil cytoplasmic autoantibody) vasculitis patient treated with RTX described the developement of mild COVID-19 symptoms (52).

A Spanish retrospective observational study cohort included patients with inflammatory rheumatic diseases receiving the following bDMARDs: TNFi (Adali- 
mumab, Certolizumab pegol, Etanercept, Golimumab, and Infliximab), IL-6 inhibitors (Interleukin-6 inhibitors Sarilumab and Tocilizumab), Abatacept, IL-17 inhibitors (Ixekizumab and Secukinumab), anti-IL-23 therapy (Ustekinumab), IL-1 inhibitors (Anakinra and Canakinumab), anti-BAFF/BLyS therapy (anti-B Lymphocyte Stimulator, Belimumab) and RTX. The study cohort also included patients treated with JAKi (Baricitinib). Patients who were treated with IL-1 inhibitors, IL-6 inhibitors, JAKi, and Belimumab demonstrated a low frequency of COVID-19 among adult patients with inflammatory rheumatic diseases (53). In addition, it was indicated that anti-IL-6 treatment may have a protective effect against COVID-19. Overall, patients with inflammatory rheumatic conditions treated with bDMARDs did not demonstrate an increased risk of SARS-CoV-2 infection or a more severe COVID-19 course (53).

Conflict of interest: none declared

Financial support: none declared

\section{REFERENCES}

1. Payne S. Family Coronaviridae. In: Payne SBT-V, ed. Viruses. Elsevier; 2017:149-158.

2. Chathappady House NN, Palissery S, Sebastian H. Corona Viruses: A Review on SARS, MERS and COVID-19. Microbiol Insights. 2021;14:117863612110024.

3. Cui J, Li F, Shi ZL. Origin and evolution of pathogenic coronaviruses. Nat Rev Microbiol. 2019;17(3):181-192.

4. Al-Tawfiq JA, Memish ZA. Drivers of MERS-CoV transmission: What do we know? Expert Rev Respir Med. 2016;10(3):331-338.

5. Cascella M, Rajnik M, Cuomo A, Dulebohn SC, Di Napoli R. Features, Evaluation, and Treatment of Coronavirus. StatPearls. 2020;(Cdc):19.

6. D'Silva KM, Wallace ZS. COVID-19 and Disease-Modifying Antirheumatic Drugs. Curr Rheumatol Rep. 2021 Apr 24;23(5):28.

7. Tariq S, Van Eeden C, Tervaert JWC, Osman MS. COVID-19, rheumatic diseases and immune dysregulation - a perspective. Clin Rheumatol. 2021;40(2):433-442.

8. Pope JE. What Does the COVID-19 Pandemic Mean for Rheumatology Patients? Curr Treat Options Rheumatol. 2020;6(2):71-74.

9. Hyrich KL, Machado PM. Rheumatic disease and COVID-19: epidemiology and outcomes. Nat Rev Rheumatol. 2021;17(2):71-72.

10. Stradner MH, Dejaco C, Zwerina J, Fritsch-Stork RD. Rheumatic Musculoskeletal Diseases and COVID-19 A Review of the First 6 Months of the Pandemic. Front Med. 2020;7(October):1-17.

11. Hu B, Guo H, Zhou P, Shi ZL. Characteristics of SARS-CoV-2 and COVID-19. Nat Rev Microbiol. 2021;19(3):141-154.

12. Park JK, Lee EB, Shin K, et al. COVID-19 Vaccination in Patients with Autoimmune Inflammatory Rheumatic Diseases: Clinical Guidance of the Korean College of Rheumatology. J Korean Med Sci. 2021;36(12):1-12.

13. Kwiatkowska B, Raciborski F, Kłak A, Mälińska M, Gryglewicz J. Early diagnosis of rheumatic diseases: An evaluation of the present situation and proposed changes. Reumatologia. 2015;53(1):3-8.

14. Boekel L, Hooijberg F, van Kempen ZLE, Vogelzang EH, Tas SW, Killestein J, et al. Perspective of patients with autoimmune diseases on COVID-19 vaccination. Lancet Rheumatol. 2021 Apr;3(4):e241-e243.

15. Calabrese L, Winthrop KL. Rheumatology and COVID-19 at 1 year: Facing the unknowns. Ann Rheum Dis. 2021;80(6):679-681.

16. Saadoun D, Vieira M, Vautier M, et al. SARS-CoV-2 outbreak in immune-mediated inflammatory diseases: the Euro-

\section{CONCLUSIONS}

The COVID-19 pandemic continues to pose notable challenges to researchers as well as clinicians. Patients with chronic inflammatory rheumatic diseases may be regarded as immunosuppressed due to their diagnosis (immune-inflammatory disease pathomechanisms frequently leading to an impairment of immune responses), as well as the medication used to treat these conditions. It has been hypothesized that both symptomatic (NSAIDs, glucocorticoids) and DMARD treatment could impact patient outcomes in the context of COVID-19. Nevertheless, data regarding the risk of developing COVID-19 and its complications in these cases remain discrepant. There is a current need of further extensive research regarding the relationship between therapy and COVID-19-related outcomes in patients with immune-inflammatory rheumatic diseases.

COVIMID multicentre cross-sectional study. Lancet Rheumatol. 2021;9913(21):1-8.

17. Conway R, Konig MF, Graef ER, Webb K, Yazdany J, Kim AHJ. Inflammatory arthritis in patients with COVID-19. Transl Res. 2021;232(January):49-59.

18. Esatoglu SN, Tascilar K, Babaoğlu H, et al. COVID-19 Among Patients With Inflammatory Rheumatic Diseases. Front Immunol. 2021;12(April):1-9.

19. Doran MF, Crowson CS, Pond GR, O'Fallon WM, Gabriel SE Frequency of infection in patients with rheumatoid arthritis compared with controls: A population-based study. Arthritis Rheum. 2002;46(9):2287-2293.

20. Gianfrancesco $\mathrm{M}$, Hyrich $\mathrm{KL}$, Hyrich $\mathrm{KL}$, et al. Characteristics associated with hospitalisation for COVID-19 in people with rheumatic disease: Data from the COVID-19 Global Rheumatology Alliance physician-reported registry. Ann Rheum Dis. 2020;79(7):859-866.

21. Listing J, Gerhold K, Zink A. The risk of infections associated with rheumatoid arthritis, with its comorbidity and treatment. Rheumatol (United Kingdom). 2013;52(1):53-61.

22. Rodriguez T. Q \& A : Overview of COVID-19 in Patients With Autoimmune Diseases. Published online 2021:1-7.

23. Ungaro RC, Agrawal M, Park S, et al. Autoimmune and Chronic Inflammatory Disease Patients with COVID-19. ACR Open Rheumatol. 2021;3(2):111-115.

24. Mikuls TR, Johnson SR, Fraenkel L, et al. American College of Rheumatology Guidance for the Management of Rheumatic Disease in Adult Patients During the COVID-19 Pandemic: Version 1. Arthritis Rheumatol. 2020;72(8):1241-1251.

25. Ladani AP, Loganathan M, Danve A. Managing rheumatic diseases during COVID-19. Clin Rheumatol. 2020;39(11):3245-3254.

26. Rezus E. Reumatologie Curs. Editura „Gr.T.Popa” UMF 2014.

27. Strangfeld A, Schäfer M, Gianfrancesco MA, et al. Factors associated with COVID-19-related death in people with rheumatic diseases: results from the COVID-19 Global Rheumatology Alliance physician-reported registry. Ann Rheum Dis. Published online 2021:930-942.

28. Haberman R, Axelrad J, Chen A, Castillo R, Yan D, Izmirly P, Neimann A, Adhikari S, Hudesman D, Scher JU. COVID-19 in Immune-Mediated Inflammatory Diseases - Case Series from New York. N Engl J Med. 2020 Jul 2;383(1):85-88.

29. Fang L, Karakiulakis G, Roth M. Are patients with hypertension and diabetes mellitus at increased risk for COVID-19 infection? Lancet Respir Med. 2020;8(4):e21. 
30. Voiriot G, Philippot Q, Elabbadi A, Elbim C, Chalumeau M, Fartoukh M. Risks Related to the Use of Non-Steroidal Anti-Inflammatory Drugs in Community-Acquired Pneumonia in Adult and Pediatric Patients. J Clin Med. 2019;8(6):786.

31. Roongta R, Ghosh A. Managing rheumatoid arthritis during COVID-19. Clin Rheumatol. 2020;39(11):3237-3244.

32. Capuano A, Scavone C, Racagni G, Scaglione F. NSAIDs in patients with viral infections, including Covid-19: Victims or perpetrators? Pharmacol Res. 2020;157(April):104849.

33. Strangfeld A, Schäfer M, Gianfrancesco MA, et al. Factors associated with COVID-19-related death in people with rheumatic diseases: Results from the COVID-19 Global Rheumatology Alliance physician-reported registry'. Ann Rheum Dis. Published online 2021:1-13.

34. Russell CD, Millar JE, Baillie JK. Clinical evidence does not support corticosteroid treatment for 2019-nCoV lung injury. Lancet. 2020;395(10223):473-475.

35. Price $E$, MacPhie $E$, Kay L, et al. Identifying rheumatic disease patients at high risk and requiring shielding during the COVID-19 pandemic. Clin Med J R Coll Physicians London. 2020;20(3).

36. Rosenbaum JT, Weisman MH, Shafer C, et al. Correspondence on "Factors associated with COVID-19-related death in people with rheumatic diseases: Results from the COVID-19 Global Rheumatology Alliance physician-reported registry." Ann Rheum Dis. Published online 2021:1-13.

37. Ahmed S, Gasparyan AY, Zimba O. Comorbidities in rheumatic diseases need special consideration during the COVID-19 pandemic. Rheumatol Int. 2021;41(2):243-256.

38. Shadmanfar S, Jonaidi-Jafari N, Jafari R, Rastgar-Mogaddam Z, Saburi A. COVID-19 in rheumatoid arthritis cases: an Iranian referral center experience. Clin Rheumatol. 2021;40(7):2979-2984.

39. D'Antiga L. Coronaviruses and Immunosuppressed Patients: The Facts During the Third Epidemic. Liver Transplant. 2020;26(6):832834

40. Martelli Júnior H, Marques NP, Marques NCT, Gomes De Lucena E, Martelli DRB, Oliveira EA. Correspondence on "Clinical course of coronavirus disease 2019 (COVID-19) in a series of 17 patients with systemic lupus erythematosus under long-term treatment with hydroxychloroquine." Ann Rheum Dis. 2021;79(6):2019-2021.

41. Wallace B, Washer L, Marder W, Kahlenberg JM. Patients with lupus with COVID-19: University of Michigan experience. Ann Rheum Dis. 2021;80(3):2021.
42. Guan W, Ni Z, Hu Y, et al. Clinical Characteristics of Coronavirus Disease 2019 in China. N Engl J Med. 2020;382(18):1708-1720.

43. Isaacs JD BG. Smart battles: immunosuppression versus immunomodulation in the inflammatory RMDs. Ann Rheum Dis. Published online 2020:79:991-3.

44. Alzahrani ZA, Alghamdi KA, Almaqati AS. Clinical characteristics and outcome of COVID-19 in patients with rheumatic diseases. Rheumatol Int. 2021;41(6):1097-1103.

45. Group RC, Horby P, LimWS, Emberson JR, MafhamM, Bell JL, Linsell $\mathrm{L}$, et al. Dexamethasone in hospitalized patients with COVID-19. N Engl J Med. Published online 2020:preliminary report.

46. Channappanavar R, Fehr AR, Vijay R, Mack M, Zhao J, Meyerholz DK PS. Dysregulated type I interferon and inflammatory monocytemacrophage responses cause lethal pneumonia in SARS-CoVinfected mice. Cell Host Microbe. 2016;19:181-193.

47. Haga S, Yamamoto N, Nakai-Murakami C, Osawa Y, Tokunaga K, Sata T, Yamamoto N, Sasazuki T, Ishizaka Y. Modulation of TNFalpha-converting enzyme by the spike protein of SARS-CoV and ACE2 induces TNF-alpha production and facilitates viral entry. Proc Natl Acad Sci U S A. 2008 Jun 3;105(22):7809-14.

48. Feldmann M, Maini RN, Woody JN, Holgate ST, Winter G, Rowland M, Richards D HT. Trials of anti-tumour necrosis factor therapy for COVID-19 are urgently needed. Lancet. Published online 2020:395:1407-1409.

49. Baker D, Roberts CAK, Pryce G, Kang AS, Marta M, Reyes S, Schmierer K, Giovannoni G AS. COVID-19 vaccine- readiness for anti-CD20-depleting therapy in autoimmune diseases. Clin Exp Immunol. Published online 2020.

50. Back I. Rituximab Associated With More Severe COVID-19 in Patients With Inflammatory Rheumatic and Musculoskeletal Diseases TOPICS : Published online 2021:1-5.

51. Avouac J, Drumez E, Hachulla E, et al. COVID-19 outcomes in patients with inflammatory rheumatic and musculoskeletal diseases treated with rituximab: a cohort study. Lancet Rheumatol. 2021;(II):419-426.

52. Suárez-Díaz S, Morán-Castaño $C$, Coto-Hernández R, MozoAvellaneda L, Suárez-Cuervo C, Caminal-Montero L. Mild COVID-19 in ANCA-associated vasculitis treated with rituximab. Ann Rheum Dis. 2020 Aug 7:annrheumdis-2020-218246.

53. Santos CS, Férnandez XC, Moriano Morales C, et al. Biological agents for rheumatic diseases in the outbreak of COVID-19: Friend or foe? RMD Open. 2021;7(1):1-7. 